\title{
Collaboration of Smart City Services with Appropriate Resource Management and Privacy Protection
}

\author{
Dr. Subarna Shakya, \\ Professor, Department of Electronics and Computer Engineering \\ Central Campus, Institute of Engineering, Pulchowk \\ Tribhuvan University, Pulchowk \\ Lalitpur Nepal - 44600 \\ Email: drss@ioe.edu.np
}

\begin{abstract}
Smart city is a quickly developing approach that is powered by Internet of Things (IoTs), providing a number of services such as collaborative diagnosis and intelligent transportation. In general, in a smart city, the terminals have certain limitations that crib their capability of processing cross application and diversified services. Due to insufficient availability of resources that can be used to develop a collaborative smart city services, a novel methodology that is highly recommended is edge computing which holds facility with high processing ability in the city terminals. However, the threat of privacy and safety of information in the collaborative services is crucial in order to ensure a safer environment of edge computing. To address this privacy issue, we have proposed an offloading method that can be used in smarty city to strengthen the privacy, promote edge utility and improve offloading efficiency. In order to establish balance between the collaborative service and privacy preservation, edge computing is integrated with information entropy. The performance is further verified using simulation analysis in appropriate environment.
\end{abstract}

Keywords: Smart city, privacy protection, internet of things, edge computing, data entropy

\section{Introduction}

Taking advantage of the advancement in the field of internet of things, cities have been in using sensors and connected devices in order to handle and collect information. By properly utilizing and processing the large amount of information generated by the IoT, a wide range of collaborative services such as elderly assistance, smart campus, intelligent medical 
Journal of Ubiquitous Computing and Communication Technologies (UCCT) (2021)

Vol.03/ No.01

Pages: 43-51

https://www.irojournals.com/jucct/

DOI: https://doi.org/10.36548/jucct.2021.1.005

diagnosis etc have been developed [1]. This also quickens the aspect of building a smart city. According to the collaborative services, it establishes improvement in visitors' QoE (quality of experience) and facilitation of residents' life using the reduced squandering resources of the city. Moreover, these smart cities are also fitted with the ability to make adjustment, understand and monitor the citizens. Because of the excessive growth rate in data generation which is further depreciated by the limited computing resources that depend completely on the IoT devices, it will result in delay of time in order to accomplish the collaborative services [2]. Hence in order to improve the quality of service (QoS) in the smart cities, there is need for resources that are connected externally, such that it improves the computing ability of the networks used. This is where edge computing comes into picture by completely utilizing the resources [3] that are available, making it capable to cater to real-time issues and have been observed to be useful in providing the apt solution to address the issues of demand for computing resources.

A number of edge nodes are deployed near the buildings and at the road sides, providing extra resources for city terminals [4]-[5]. Using the low end-to-end communication latency and richer computing resources, it is possible to use this methodology to decrease the response time by a large extent. If an overload occurs at the edge note, the requests on computing from the city terminals will not be taken and there will also be a hold up in service till the required resources are accessible. Because of this, the service that is in the queue will be transferred to another edge node, saving waiting time in the queue. Thus it is essential to take into consideration the queuing time that is possible in service offloading such that a proper balance is stroked between offloading and queuing. Despite these preventive measures [6], there is still possibility of private information being disclosed to third parties, making it a major threat in offloading collaborative services. Information that is labelled as private or sensitive is initially sent to the edge nodes from the terminals in the smart city and the result is then processed to be returned by the server. At the time of offloading service, leakage of data in an unexpected turn of events will prove to be fatal. Moreover, since information is passed through a number of devices, it is not possible to guarantee security of this information [7]. 
Journal of Ubiquitous Computing and Communication Technologies (UCCT) (2021)

Vol.03/ No.01

Pages: 43-51

https://www.irojournals.com/jucct/

DOI: https://doi.org/10.36548/jucct.2021.1.005

In general, collaborative services are used to hold and transact sensitive information which will prevent harm to the data that might have occurred through ENs [8]. Since there is a number of ENs that are located in the smart city, resources wastage should be avoided and their efficiency should also be improved by establishing load balance [9]. Moreover, in order to realise a sustainable development of smart cities, the consumption of energy by the edge nodes have to be optimized. Apart from edge utility comprising of energy consumption and load balance, one should also take into consideration the offloading efficiency [10] to decrease service response time thereby improving quality of service. But, the difficulty in attaining this is the trade off between maintaining load balance, improving energy consumption and decreasing service response time while at the same time ensuring security of data [11]. The main contributions of this work can be briefed as given below:

- To establish queuing model, the average waiting time of the EN can be elaborated and analyzed using the $\mathrm{M} / \mathrm{M} / \mathrm{Z} / \infty / \infty$ methodology.

- The privacy analysis, the edge utility and the offloading efficiency are modelled with respect to the collaborative services of smart city.

- To attain the goal of improved service response time, decrease load balance and minimized energy consumption, an intelligent offloading technique is introduced.

- Experimental analysis is carried out and compared with other similar methodologies to establish the efficiency and effectiveness of the proposed work.

The rest of the paper is organized such that Section II gives a detailed view of the proposed model. Section III explains analyzes and compares the results that are observed and Section IV concludes the work based on the findings recorded and compared. 


\section{Proposed Work}

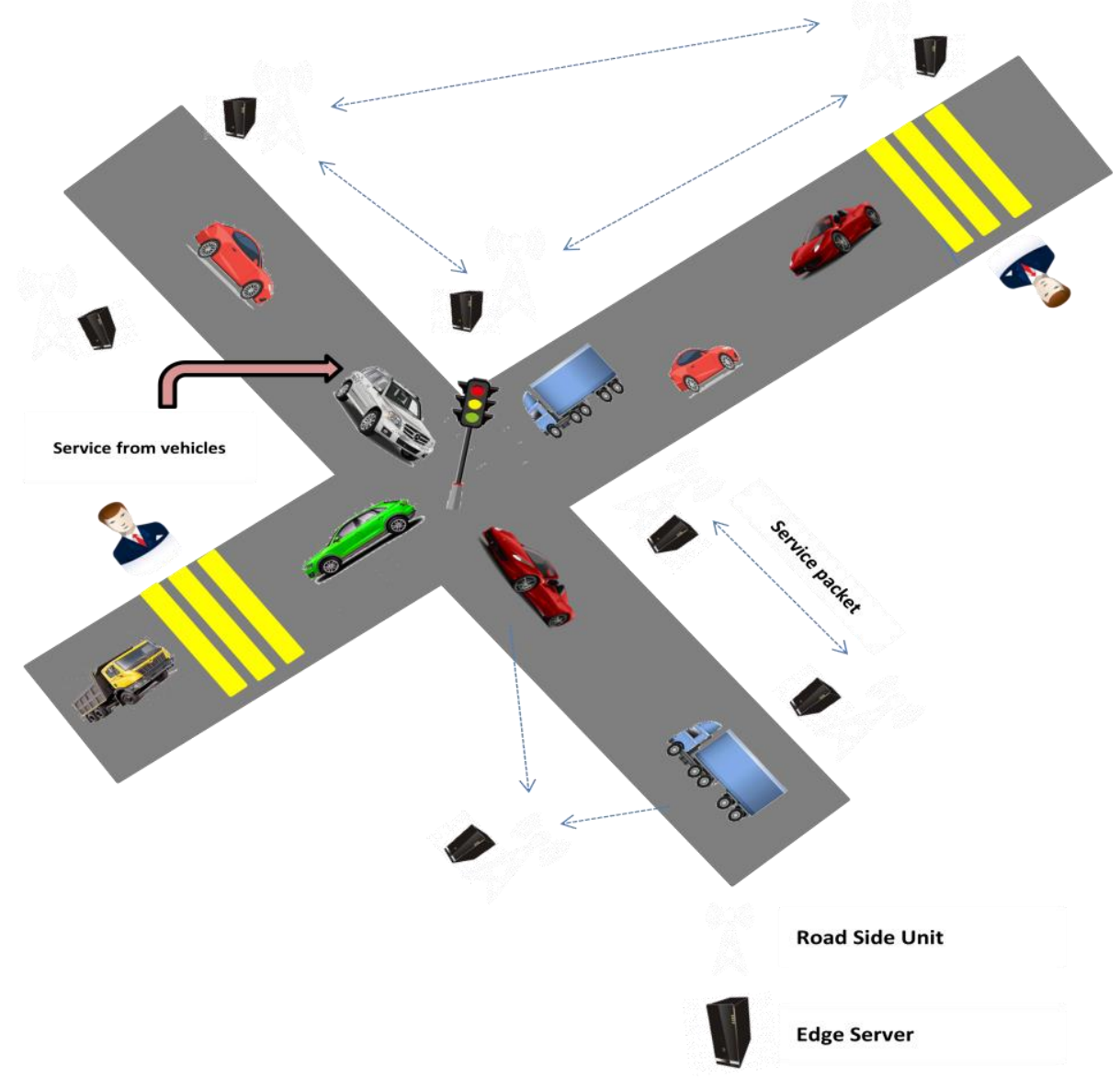

Fig.1. Edge Computing in a Collaborative Smart City

Fig.1 shows the offloading framework of a collaborative smart city service using edge computing [12]. It comprises of a unit along the road side with the ability to receive information and an edge server that can communicate with the receiver. A total of ' $\mathrm{M}$ ' edge nodes are used in this proposed work that can be represented as $\mathrm{R}=\left\{r_{1}, r_{2}, \ldots, r_{M}\right\}$. There is need for service sets that is provided to the terminal that requires offloading as $S=\left\{s_{1}, s_{2}, \ldots, s_{E}\right\}$. The terminals of the city are distributed in a random fashion and the terminal set is represented as $\mathrm{E}=\left\{e_{1}, e_{2}, \ldots, e_{E}\right\}$. The computation based smart city services require quick feedback and the city terminals will provide this to the edge nodes. Consider a binary variable $x_{E, N} \in\{0,1\}$ such that if it takes the value of 1 , the service is sent from the terminal to the ED, else there is no connection between the two points [13]. 
Journal of Ubiquitous Computing and Communication Technologies (UCCT) (2021)

Vol.03/ No.01

Pages: 43-51

https://www.irojournals.com/jucct/

DOI: https://doi.org/10.36548/jucct.2021.1.005

In this proposed work, we have incorporated execution time, transmission time and waiting time in the service response time. If $\mathrm{Z}$ represents the total number of virtual machines (VMs) in an edge node, then according to queuing theory, we can use Poisson distribution to determine the collaborative smart city services. $\gamma$ represents the Poisson distribution parameter and $\beta$ indicates the VM parameter. Based on the proposed $\mathrm{M} / \mathrm{M} / \mathrm{Z} / \infty / \infty$ queuing methodology, it is possible to determine the service intensity of VM using the formula:

$$
\xi=\frac{\gamma}{\beta}
$$

If there are $\mathrm{N}$ VMs present in an edge node, then the service intensity can be formulated as:

$$
\xi=\frac{\gamma}{N \cdot \beta} \quad\left(\xi_{N}<1\right)
$$

If there are $\mathrm{i}$ offloaded services and the probability is represented as $p_{i}^{\text {num }}$, then the probability can be calculated as

$$
p_{0}^{n u m}=\frac{1}{\sum_{m=0}^{N-1} \frac{\xi}{N !(1-\xi N)}+\frac{1}{n !} \xi m}
$$

Based on this expression, a generalized probability of the services offloaded can be represented as:

$$
p_{i}^{\text {num }}=\left\{\begin{array}{l}
\frac{\left(\frac{\gamma}{\beta}\right)^{i}}{i !} \cdot p_{0}^{\text {num }},(1 \leq \mathrm{i} \leq \mathrm{N} \\
\frac{\left(\frac{\gamma}{\beta}\right)^{i}}{N ! N^{(i-N)}} \cdot p_{0}^{\text {num }}, \quad(i>N)
\end{array}\right.
$$

An example of Ant Colony Optimization in edge computing is shown in Fig.2. In an EN, if there are lesser than $\mathrm{N}$ services offloaded, it indicates that the resources available for offloading are sufficient and there will be no queue time. In such circumstances, the city service is immediately executed when it reaches the edge node. On the other hand, if there are more offloaded services than the available VM, there will be a queue and waiting time for executing city services. This average waiting time can be represented as shown in (5)

$$
w t^{q}=\sum_{i=N+1}^{\infty}(i-N) \cdot P_{i}=\frac{\xi^{N} \cdot P_{0} \cdot \xi_{N}}{N !\left(1-\xi_{N}\right)^{2}}(i>N)
$$

The time taken to send the service to the edge node can be calculated using the formula: 
Journal of Ubiquitous Computing and Communication Technologies (UCCT) (2021)

Vol.03/ No.01

Pages: 43-51

https://www.irojournals.com/jucct/

DOI: https://doi.org/10.36548/jucct.2021.1.005

$$
t_{x}^{t r}=\sum_{i=1}^{I} a_{n, i} \cdot \frac{v_{x}}{B^{u n}}
$$

where B represents the bandwidth of the wireless channel and $v_{x}$ denotes size of data in the service. When offloading collaborative services, they are said to be transmitted to various ENs for purpose of execution. In order to prevent wastage of resources by properly balancing the resource allocation, there is need for load balancing. It is necessary to properly balance the CMs and ENs such that the occupied VMs in an EN is represented using the expression;

$$
C_{i}=\left\{\begin{array}{c}
N,\left(\sum_{i=1}^{I} a_{x, i}>N\right. \\
\sum_{i=1}^{I} a_{x, i},\left(1 \leq \sum_{i=1}^{I} a_{x, i} \leq N\right.
\end{array}\right.
$$

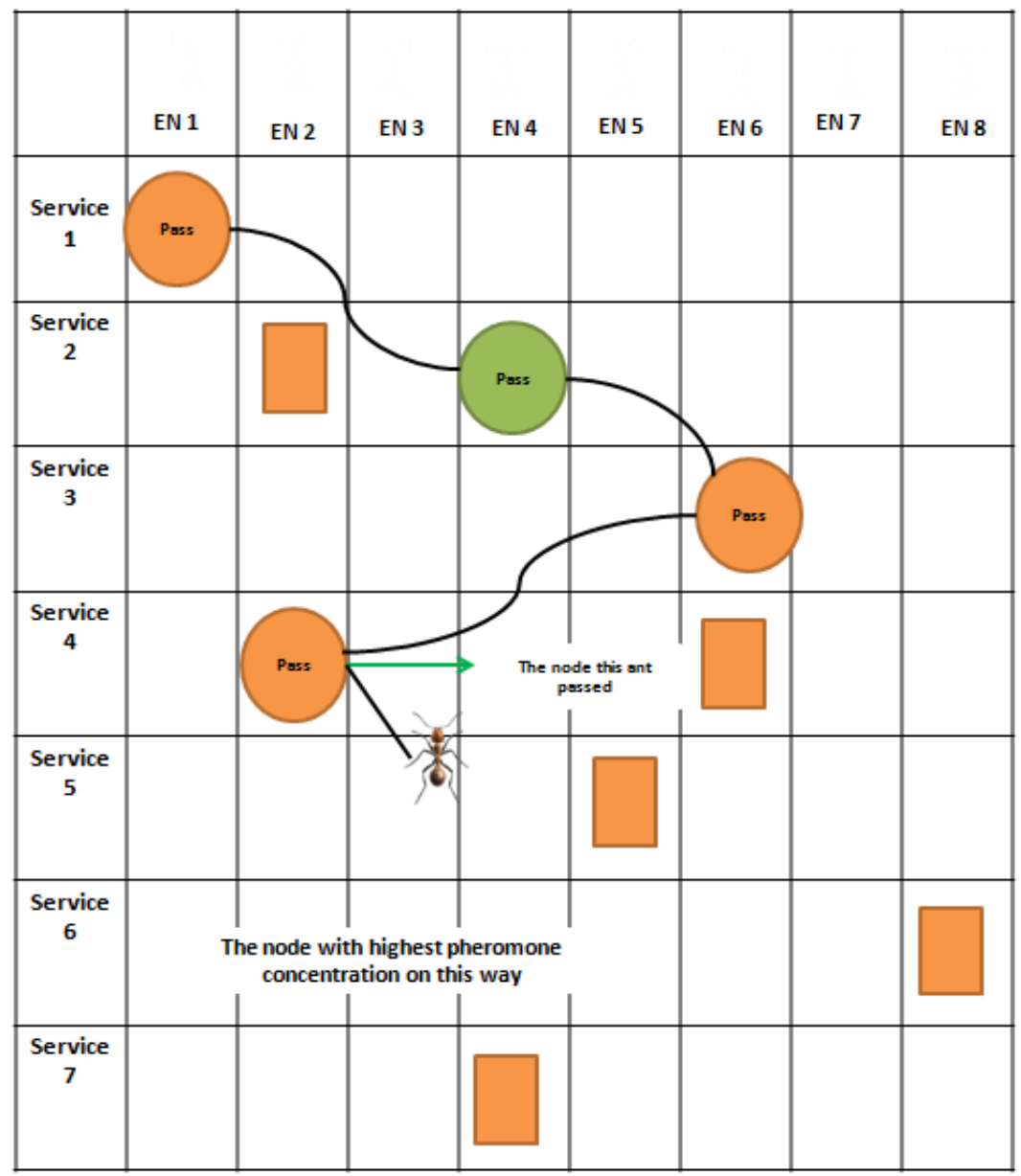

Fig.2. Ant Colony Optimization in Edge Computing 
Journal of Ubiquitous Computing and Communication Technologies (UCCT) (2021)

Vol.03/ No.01

Pages: 43-51

https://www.irojournals.com/jucct/

DOI: https://doi.org/10.36548/jucct.2021.1.005

\section{Results and Discussion}

The ACO searches for the apt solution to provide guaranteed efficiency and quick speed. In this proposed work, we have measured the outputs of the proposed work IOM with the Benchmark, First Fit (FF) and Next Fit (NF). Fig.3 shows a comparison of the ENs that are occupied. It is seen that IOM proves a higher rate of occupancy when compared with the other methodologies. Similarly in Fig.4 and Fig.5, the total amount of energy consumed and the average time for response is observed and recorded. It is noted that there is a positive increase in the proposed methodology when compared with the other methodologies.

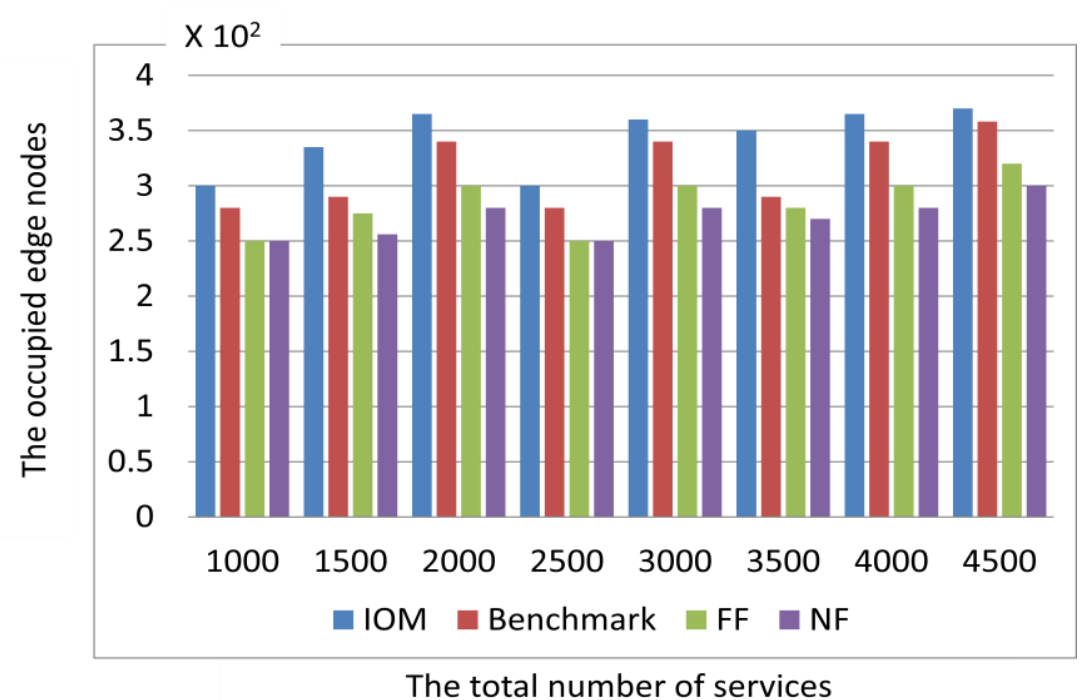

Fig.3. Occupied ENs- A comparison

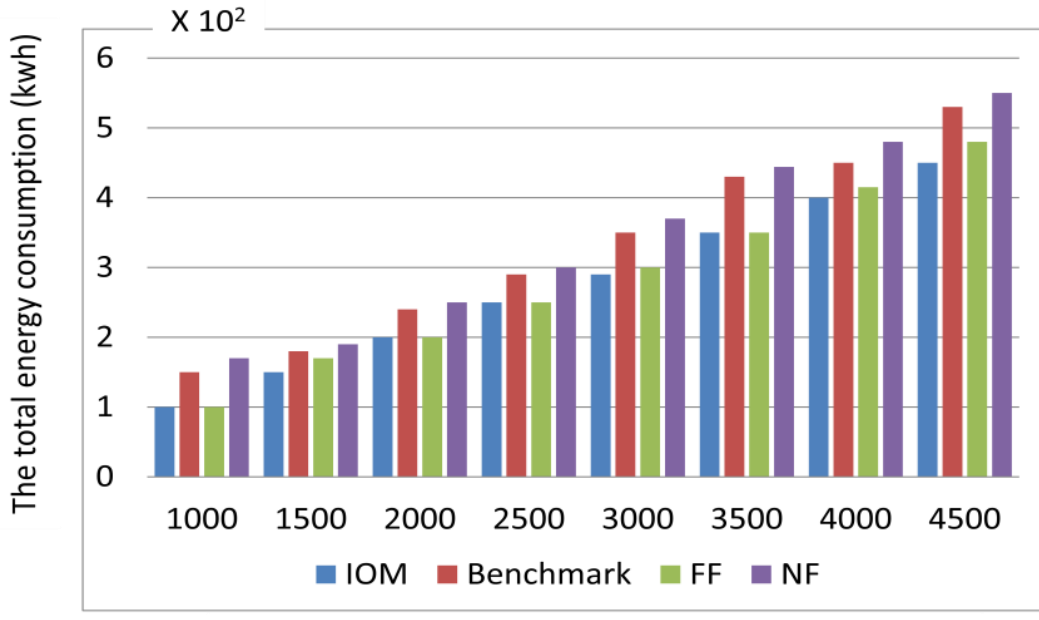

The total number of services

Fig.4. Energy Consumption 


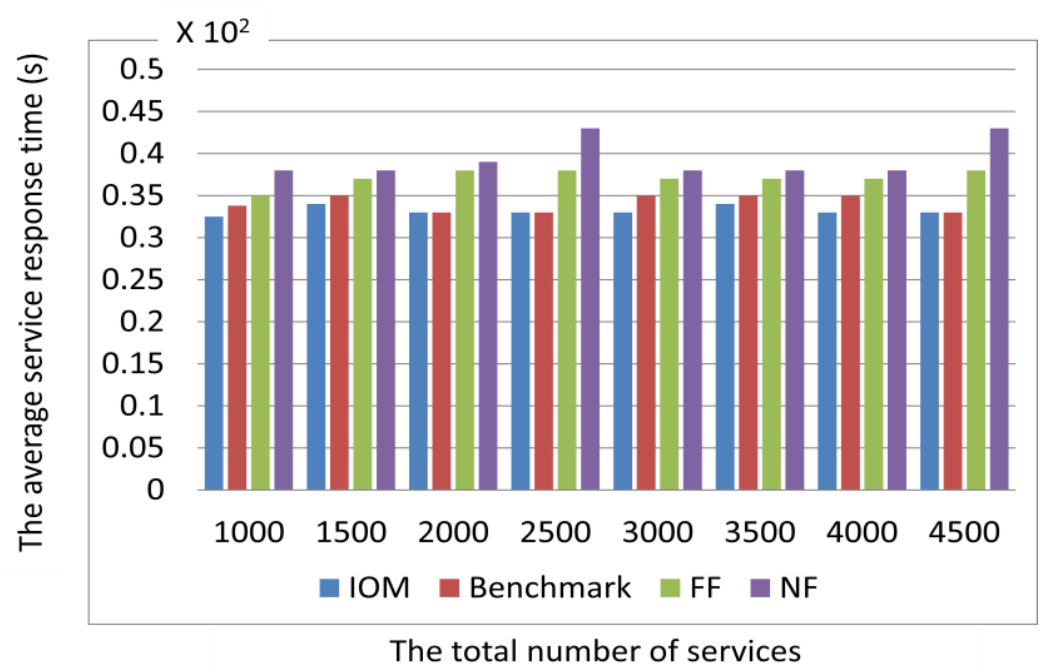

Fig.5. Average response time for a service

\section{Conclusion}

Low QoS and long service response time are some of the weaknesses of collaborative smart city that still needs some upgrading. Smart city is built on edge computing to improve the drawbacks that are observed in terminals in cities. In this proposed work, we have designed a smart city service using intelligent offloading. It uses the advantages of both the system to attain proper load balance, optimizing energy consumption and minimizing service response time. Experimental evaluation shows a significant increase in efficiency and effectiveness of the proposed work. Future work can focus on applying this methodology to real like, taking into consideration the actual information of smart city in real-time.

\section{References}

[1] Dameri, R. P. (2017). Smart city implementation. Progress in IS; Springer: Genoa, Italy.

[2] Zubizarreta, I., Seravalli, A., \& Arrizabalaga, S. (2016). Smart city concept: What it is and what it should be. Journal of Urban Planning and Development, 142(1), 04015005.

[3] Hashem, I. A. T., Chang, V., Anuar, N. B., Adewole, K., Yaqoob, I., Gani, A., ... \& Chiroma, H. (2016). The role of big data in smart city. International Journal of Information Management, 36(5), 748-758. 
Journal of Ubiquitous Computing and Communication Technologies (UCCT) (2021)

Vol.03/ No.01

Pages: 43-51

https://www.irojournals.com/jucct/

DOI: https://doi.org/10.36548/jucct.2021.1.005

[4] Smys, S., Wang, H., \& Basar, A. (2021). 5G Network Simulation in Smart Cities using Neural Network Algorithm. Journal of Artificial Intelligence, 3(01), 43-52.

[5] Al-Hader, M., \& Rodzi, A. (2009). The smart city infrastructure development \& monitoring. Theoretical and Empirical Researches in Urban Management, 4(2 (11), 87 94.

[6] Camero, A., \& Alba, E. (2019). Smart City and information technology: A review. Cities, 93, 84-94.

[7] Shirley, D. Ruth Anita. "Systematic diagnosis of power switches." In 2014 International Conference on Embedded Systems (ICES), pp. 32-34. IEEE, 2014.

[8] Chen, J. I. Z. (2019). 5G technology and advancements in connected livingcomprehensive survey. Journal of Electronics, 1(02), 71-79.

[9] Cardullo, P., Di Feliciantonio, C., \& Kitchin, R. (Eds.). (2019). The right to the smart city. Emerald Group Publishing.

[10] Ramaprasad, A., Sánchez-Ortiz, A., \& Syn, T. (2017, September). A unified definition of a smart city. In International Conference on Electronic Government (pp. 13-24). Springer, Cham.

[11] Shakya, S. (2019). Virtual restoration of damaged archeological artifacts obtained from expeditions using 3D visualization. Journal of Innovative Image Processing (JIIP), l(02), 102-110.

[12] Jucevičius, R., Patašienè, I., \& Patašius, M. (2014). Digital dimension of smart city: critical analysis. Procedia-Social and Behavioral Sciences, 156, 146-150.

[13] Bashar, D. A. (2020). Review on sustainable green Internet of Things and its application. J. Sustain. Wireless Syst, 1(4), 256-264. 